\title{
Stochastic Responses of Lightly Nonlinear Vibroimpact System with Inelastic Impact Subjected to External Poisson White Noise Excitation
}

\author{
Guidong Yang $\mathbb{D},{ }^{1}$ Wei $\mathrm{Xu}^{2}{ }^{2}$ Dongmei Huang $\mathbb{D},{ }^{1}$ and Mengli Hao ${ }^{3}$ \\ ${ }^{1}$ School of Mathematics and Statistics, Xidian University, Xian, Shaanxi 710071, China \\ ${ }^{2}$ Department of Applied Mathematics, Northwestern Polytechnical University, Xian, Shaanxi 710072, China \\ ${ }^{3}$ Department of Applied Mathematics, Xian University of Technology, Xian, Shaanxi 710048, China
}

Correspondence should be addressed to Guidong Yang; gdyang@xidian.edu.cn

Received 20 December 2017; Revised 5 March 2018; Accepted 14 March 2018; Published 24 April 2018

Academic Editor: Ivan Giorgio

Copyright (C) 2018 Guidong Yang et al. This is an open access article distributed under the Creative Commons Attribution License, which permits unrestricted use, distribution, and reproduction in any medium, provided the original work is properly cited.

A procedure for analyzing stationary responses of lightly nonlinear vibroimpact system with inelastic impact subjected to external Poisson white noise excitation is proposed. First, the original vibroimpact system is transformed to a new system without velocity jump in terms of the Zhuravlev nonsmooth coordinate transformation and the Dirac delta function. Second, the averaged generalized Fokker-Planck-Kolmogorov (FPK) equation for transformed system under parametric excitation of Poisson white noise is derived by stochastic averaging method. Third, the averaged generalized FPK equation is solved by using the perturbation technique and inverse transformation of the Zhuravlev nonsmooth coordinate transformation to obtain the approximately stationary solutions for response probability density functions of original vibroimpact system. Last, analytical and numerical results for two typical lightly nonlinear vibroimpact systems are presented to assess the effectiveness of the proposed method. It is found that they are in good agreement and the proposed method is quite effective.

\section{Introduction}

Vibroimpact system, as a class of typical nonsmooth system [1], has attracted much attention in the past few decades due to its existence in various engineering applications $[2,3]$. Certain applications or problems involved impacts include vibratory pile drivers, heat exchanger tube wear in nuclear power stations, large gaps in labyrinth seals for rotating shafts of high-pressure pumps, and a moored body with an inextensible mooring line [4-7]. There are two commonly used methods to model impacts. One is the classical or hard impact model [1], and the other is the Hertz contact model or soft impact model $[8,9]$. The classical impact model consists of two parts: the ordinary motion described by a differential equation and the impact condition. In the Hertz contact model, the contact force between two elastic bodies is assumed to be proportional to the $3 / 2$ (sometimes with different values) power of the relative displacement between them. By means of the two models, many interesting dynamical behaviors were observed such as chaos motion and bifurcations [10,11], Hopf bifurcation [12], grazing bifurcation $[13,14]$, torus bifurcation [15], chattering bifurcation $[16,17]$, sticking motion $[18,19]$, and border collision bifurcation [20].

As is known to all, random excitations widely exist in vibroimpact systems. Their effects usually can not be neglected and sometimes are even quite large [21]. Therefore, studies on the prediction of stochastic responses of vibroimpact systems are naturally important but difficult due to the presence of random excitations and nonsmooth factors. In recent years, some methods [22-27] have been developed to explore the response probability density function (PDF) which is an important characteristic of a stochastic system. For the classical impact model, Dimentberg et al. [28, 29] studied stochastic response of linear vibroimpact system under Gaussian white noise. Feng et al. [30,31] explored the response PDFs of Duffing-Van der Pol vibroimpact system under independent and correlated Gaussian white noises, respectively. Zhu [32] obtained the response PDFs of a vibroimpact Duffing system with a randomly varying damping 


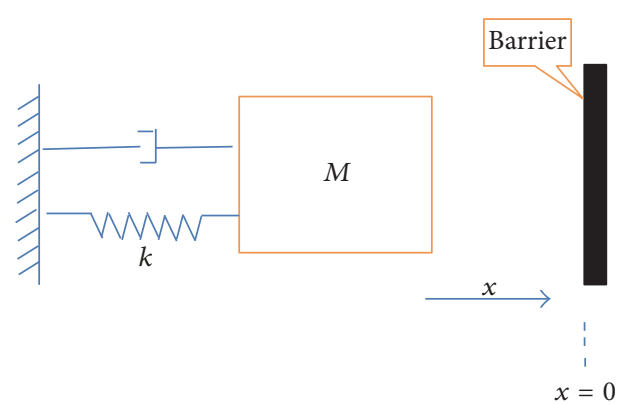

FIGURE 1: Vibroimpact system with a rigid barrier on right side.

term. For the Hertz contact model, Huang et al. [33] investigated stationary responses of multi-degree-of-freedom vibroimpact system under white noise excitations. Xu et al. [34] analyzed random vibration problems of vibroimpact system with inelastic impact. Although stochastic responses of vibroimpact system have been reported, the above studies are limited to responses of vibroimpact systems subject to Gaussian white noise. In practice, many random excitations are essentially non-Gaussian, for example, those due to sea wave [35] and wind [36]. The Poisson white noise is the most used model for non-Gaussian random excitations [37-40]. However, the research on stochastic responses of vibroimpact system subject to Poisson white noise has been rarely addressed [41] and is far from enough. This paper is devoted to presenting a procedure to predict the stochastic responses of lightly nonlinear vibroimpact system subject to external Poisson white noise excitation. In the following parts, the Zhuravlev nonsmooth coordinate transformation [22], the stochastic averaging method [42], and the perturbation technique [43] are applied in succession to obtain the stationary solutions of response PDFs. Two examples are also presented to assess the effectiveness of the procedure. Comparison between results obtained from the proposed method and those from MonteCarlo simulation shows that they are in good agreement. Therefore, the proposed procedure is quite effective.

\section{Problem Statement}

Without loss of generality, a simple mass-spring-damper model (Figure 1) for single-degree-of-freedom lightly nonlinear vibroimpact system with inelastic impact subjected to external Poisson white noise excitation is considered, and the corresponding equation of motion can be expressed as

$$
\begin{array}{rlrl}
\ddot{x}+\varepsilon^{2} f(x, \dot{x})+k x & =\varepsilon \xi(t), & & x<0, \\
\dot{x}_{+} & =-r \dot{x}_{-}, & x=0,
\end{array}
$$

where $\varepsilon>0$ is a small parameter; $\varepsilon^{2} f(x, \dot{x})$ denotes lightly linear or nonlinear damping force; $k$ is the stiffness coefficient; $\xi(t)$ is Poisson white noise; $\dot{x}_{+}$and $\dot{x}_{-}$denote the rebound and impact velocity, respectively; the subscripts "+" and "-" refer to values of velocity just before and after impact; $r$ is the restitution factor whose value is among zero and one. Particularly, when $r$ equals one, the system reduces to an elastic impact one. $x=0$ denotes that the rigid barrier is located at the system's equilibrium position. Equation (1a) governs the system's motion between impacts as $x<0$. Equation (1b) describes the impact law, which means that the oscillator's velocity has an instantaneous change when it impacts with the barrier at $x=0$.

The Poisson white noise can be treated as the formal derivative of the compound Poisson process $C(t)$; that is,

$$
\xi(t)=\frac{d C(t)}{d t}
$$

in which

$$
C(t)=\sum_{i=1}^{N(t)} Y_{i} U\left(t-t_{i}\right)
$$

where $N(t)$ is the Poisson counting process with a constant impulse arrival rate $\lambda$ which also means the expected number of event occurrences per second. $Y_{i}$ is a random variable representing the random magnitude of $i$ th impulse arriving at time $t_{i} . U(\cdot)$ is a unit step function. Assume that $Y_{i}$ are independent identically distributed random variables with zero mean which are independent of the impulse arrival time $t_{i}$. $C(t)$ is a process with independent increments, and the $k$ th correlation function of whose increment process $d C(t)=$ $C(t+d t)-C(t)$ is as follows [44]:

$$
\begin{aligned}
R^{(k)} & {\left[d C\left(t_{1}\right), d C\left(t_{2}\right), \ldots, d C\left(t_{k}\right)\right] } \\
& =\lambda E\left[Y^{k}\right] \delta\left(t_{2}-t_{1}\right) \cdots \delta\left(t_{k}-t_{1}\right) d t_{1} d t_{2} \cdots d t_{k} .
\end{aligned}
$$

By the relation between the correlation function and the moment function of random processes, omitting the small quantity that higher than $d t$, the $k$ th moment function can be derived as

$$
E\left[(d C)^{k}\right]=\lambda E\left[Y^{k}\right] d t
$$

Given that the nonsmooth characteristic of vibroimpact system described by (1a) and (1b), the following coordinate transformation introduced by Zhuravlev [22] is used as

$$
\begin{gathered}
x=x_{1}=|y|, \\
\dot{x}=x_{2}=\dot{y} \text { sgn } y, \\
\operatorname{sgn} y= \begin{cases}1, & y>0 \\
0, & y=0 \\
-1, & y<0 .\end{cases}
\end{gathered}
$$

The relation between the original variables $x, \dot{x}$ and the new variables $y, \dot{y}$ can be simply represented by Figures 2 and 3 .

Substituting (6) into (1a) and (1b), the transformation maps the domain $x<0$ of original phase plane $(x, \dot{x})$ onto the whole phase plane $(y, \dot{y})$ (see Figure 3 ). Since $(d / d y)(\operatorname{sgn} y)=0$ when $y \neq 0$, this implies also that $\ddot{x}(t)=$ $\ddot{y}(t) \operatorname{sgn} y$. When $y=0$, the impact/rebound condition for the transformed state variables is found to be $\dot{y}_{+}=r \dot{y}_{-}$ 


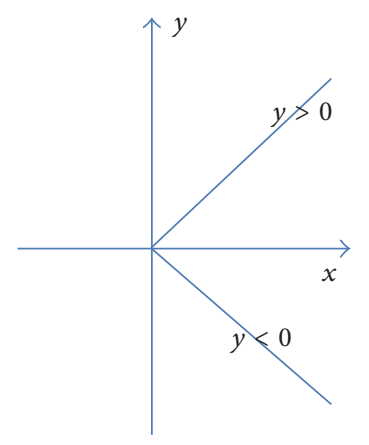

(a)

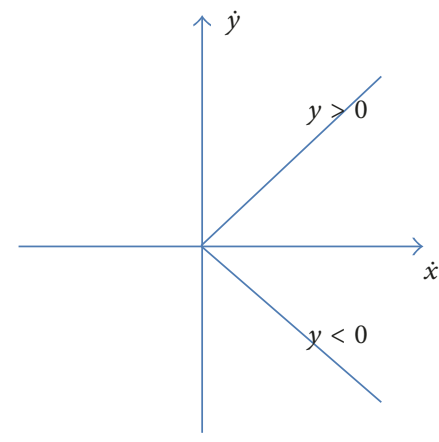

(b)

FIGURE 2: Relation between the original variables and the new variables: (a) $x$ and $y$; (b) $\dot{x}$ and $\dot{y}$.

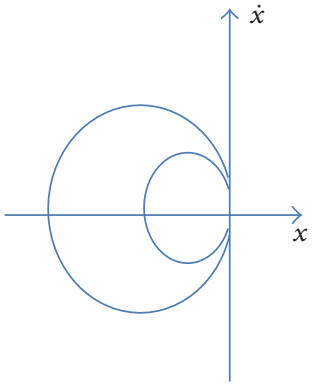

(a)

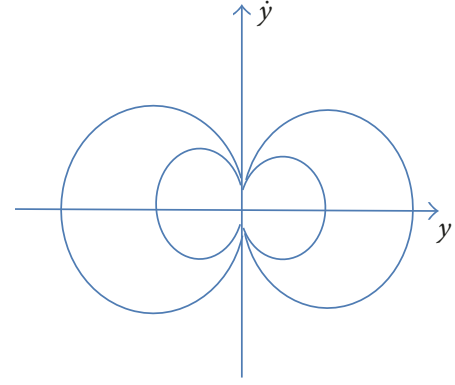

(b)

FIGURE 3: Phase plane for vibroimpact system: (a) original vibroimpact system; (b) the transformed system.

according to Dimentberg and Menyailov [28]. Note that the identity $(\operatorname{sgn} y)^{2}=1$, and the original equation of motion can be replaced by

$$
\begin{array}{r}
\ddot{y}+\varepsilon^{2} f(y \operatorname{sgn} y, \dot{y} \operatorname{sgn} y) \operatorname{sgn} y+k y=\varepsilon \operatorname{sgn} y \xi(t), \\
t \neq t_{*}, \\
\dot{y}_{+}=r \dot{y}_{-}, \quad t=t_{*},
\end{array}
$$

where $t_{*}$ represents the time instant of impact corresponding to $y=0$, that is, $y\left(t_{*}\right)=0$. Also, the velocity jump becomes proportional to $1-r$ instead of $1+r$ for original one. Using the Dirac delta function, an additional impulsive term can be introduced as follows:

$$
\left(\dot{y}_{-}-\dot{y}_{+}\right) \delta\left(t-t_{*}\right)=(1-r) \dot{y}|\dot{y}| \delta(y) .
$$

According to [28], it is reasonable to treat (7b) as an additional impulse of (7a) as $t=t_{*}$. Therefore, (7a), (7b), and (8) can be combined into one new equation as

$$
\begin{aligned}
\ddot{y}+\varepsilon^{2} f(y \operatorname{sgn} y, \dot{y} \operatorname{sgn} y) \operatorname{sgn} y+k y \\
\quad-(r-1) \dot{y}|\dot{y}| \delta(y)=\varepsilon \operatorname{sgn} y \xi(t) .
\end{aligned}
$$

The above transformed equation may permit rigorous analytical study since there contains no velocity jump and the impact condition is taken place by an additional impulsive term.

\section{Stochastic Responses}

3.1. Stochastic Averaging. When the values of $(1-r)$ approaches to zero, the energy loss of impact is very small, and the system becomes a quasiconservative one as long as the damping coefficients and random excitations are weak. Therefore, the stochastic averaging method can be applied to study the response of the transformed system analytically. For simplicity, $(1-r)$ is assumed of order $o\left(\varepsilon^{2}\right)$. The transformed equation (9) is equivalent to a pair of first-order equations:

$$
\begin{aligned}
d y_{1}= & y_{2} d t, \\
d y_{2}= & -\varepsilon^{2} \tilde{f}\left(y_{1}, y_{2}\right)-k y_{1}+(r-1) y_{2}\left|y_{2}\right| \delta\left(y_{1}\right) \\
& +\varepsilon \operatorname{sgn} y_{1} d C(t),
\end{aligned}
$$

where

$$
\tilde{f}\left(y_{1}, y_{2}\right)=f\left(y_{1} \operatorname{sgn} y_{1}, y_{2} \operatorname{sgn} y_{1}\right) \operatorname{sgn} y_{1} .
$$

The total energy of system is

$$
H=\frac{1}{2} y_{2}^{2}+\frac{1}{2} k y_{1}^{2}
$$


Substituting (12) into (10) yields the following Stratonovich stochastic differential equations about displacement $y_{1}$ and energy $H$ with parametric excitation of Poisson white noise:

$$
\begin{aligned}
& d y_{1}=\frac{\partial H}{\partial y_{2}} d t \\
& d H=-\varepsilon^{2} \tilde{f}\left(y_{1}, \frac{\partial H}{\partial y_{2}}\right) \frac{\partial H}{\partial y_{2}} d t
\end{aligned}
$$

$$
\begin{aligned}
& +(r-1)\left(\frac{\partial H}{\partial y_{2}}\right)^{2}\left|\frac{\partial H}{\partial y_{2}}\right| \delta\left(y_{1}\right) d t \\
& +\varepsilon \operatorname{sgn} y_{1} \frac{\partial H}{\partial y_{2}} \circ d C(t) .
\end{aligned}
$$

The corresponding Itô stochastic differential equations are derived using the converting rule for systems with Poisson white noise excitation proposed by Di Paola and Falsone [37]:

$$
\begin{aligned}
d y_{1}= & \frac{\partial H}{\partial y_{2}} d t \\
d H= & -\varepsilon^{2} \tilde{f}\left(y_{1}, \frac{\partial H}{\partial y_{2}}\right) \frac{\partial H}{\partial y_{2}} d t+(r-1)\left(\frac{\partial H}{\partial y_{2}}\right)^{2}\left|\frac{\partial H}{\partial y_{2}}\right| \delta\left(y_{1}\right) d t+\varepsilon \operatorname{sgn} y_{1} \frac{\partial H}{\partial y_{2}} d C(t) \\
& +\frac{\varepsilon^{2}}{2 !}\left(\operatorname{sgn} y_{1} \frac{\partial H}{\partial y_{2}}\right) \frac{\partial}{\partial H}\left(\operatorname{sgn} y_{1} \frac{\partial H}{\partial y_{2}}\right)[d C(t)]^{2} \\
& +\frac{\varepsilon^{3}}{3 !}\left(\operatorname{sgn} y_{1} \frac{\partial H}{\partial y_{2}}\right) \frac{\partial}{\partial H}\left[\left(\operatorname{sgn} y_{1} \frac{\partial H}{\partial y_{2}}\right) \frac{\partial}{\partial H}\left(\operatorname{sgn} y_{1} \frac{\partial H}{\partial y_{2}}\right)\right][d C(t)]^{3} \\
& +\frac{\varepsilon^{4}}{4 !}\left(\operatorname{sgn} y_{1} \frac{\partial H}{\partial y_{2}}\right) \frac{\partial}{\partial H}\left\{\left(\operatorname{sgn} y_{1} \frac{\partial H}{\partial y_{2}}\right) \frac{\partial}{\partial H}\left[\left(\operatorname{sgn} y_{1} \frac{\partial H}{\partial y_{2}}\right) \frac{\partial}{\partial H}\left(\operatorname{sgn} y_{1} \frac{\partial H}{\partial y_{2}}\right)\right]\right\}[d C(t)]^{4}+\cdots
\end{aligned}
$$

Give that

$$
\begin{gathered}
\left(\operatorname{sgn} y_{1} \frac{\partial H}{\partial y_{2}}\right) \frac{\partial}{\partial H}\left(\operatorname{sgn} y_{1} \frac{\partial H}{\partial y_{2}}\right) \\
=\frac{1}{2} \frac{\partial}{\partial H}\left[\left(\operatorname{sgn} y_{1} \frac{\partial H}{\partial y_{2}}\right)^{2}\right]=1 .
\end{gathered}
$$

Equation (14) can be simplified as

$$
\begin{aligned}
d y_{1}= & \frac{\partial H}{\partial y_{2}} d t \\
d H= & -\varepsilon^{2} \widetilde{f}\left(y_{1}, \frac{\partial H}{\partial y_{2}}\right) \frac{\partial H}{\partial y_{2}} d t \\
& +(r-1)\left(\frac{\partial H}{\partial y_{2}}\right)^{2}\left|\frac{\partial H}{\partial y_{2}}\right| \delta\left(y_{1}\right) d t \\
& +\varepsilon \operatorname{sgn} y_{1} \frac{\partial H}{\partial y_{2}} d C(t)+\frac{\varepsilon^{2}}{2 !}[d C(t)]^{2} .
\end{aligned}
$$

The generalized FPK equation associated with (16) is

$$
\begin{aligned}
\frac{\partial \rho}{\partial t} & =-\frac{\partial}{\partial y_{1}}\left(\frac{\partial h}{\partial y_{2}} \rho\right)-\frac{\partial}{\partial h}\left\{\left(-\varepsilon^{2} \tilde{f}\left(y_{1}, \frac{\partial h}{\partial y_{2}}\right) \frac{\partial h}{\partial y_{2}}\right.\right. \\
& +(r-1)\left(\frac{\partial h}{\partial y_{2}}\right)^{2}\left|\frac{\partial h}{\partial y_{2}}\right| \delta\left(y_{1}\right)+\varepsilon \operatorname{sgn} y_{1} \frac{\partial h}{\partial y_{2}} E[Y]
\end{aligned}
$$

$$
\begin{aligned}
& \left.\left.+\frac{\varepsilon^{2}}{2} \lambda E\left[Y^{2}\right]\right) \rho\right\}+\frac{1}{2 !} \frac{\partial^{2}}{\partial h^{2}}\left\{\left(\varepsilon^{2}\left(\frac{\partial h}{\partial y_{2}}\right)^{2} \lambda E\left[Y^{2}\right]\right.\right. \\
& \left.\left.+\varepsilon^{3}\left(\operatorname{sgn} y_{1}\right)^{3} \frac{\partial h}{\partial y_{2}} \lambda E\left[Y^{3}\right]+\frac{\varepsilon^{4}}{4} \lambda E\left[Y^{4}\right]\right) \rho\right\}-\frac{1}{3 !} \\
& \cdot \frac{\partial^{3}}{\partial h^{3}}\left\{\left(\varepsilon^{3}\left(\operatorname{sgn} y_{1}\right)^{3}\left(\frac{\partial h}{\partial y_{2}}\right)^{3} \lambda E\left[Y^{3}\right]\right.\right. \\
& \left.\left.+\frac{3}{2} \varepsilon^{4}\left(\frac{\partial h}{\partial y_{2}}\right)^{2} \lambda E\left[Y^{4}\right]\right) \rho\right\}+\frac{1}{4 !} \frac{\partial^{4}}{\partial h^{4}}\left\{\varepsilon^{4}\left(\frac{\partial h}{\partial y_{2}}\right)^{4}\right. \\
& \left.\cdot \lambda E\left[Y^{4}\right] \rho\right\}+O\left(\varepsilon^{5}\right),
\end{aligned}
$$

where $\rho=\rho\left(y_{1}, h, t\right)$.

Particularly, if the probability distribution of $Y$ is symmetric, all odd order moments are zero. Thus, we have

$$
\begin{aligned}
\frac{\partial \rho}{\partial t} & =-\frac{\partial}{\partial y_{1}}\left(\frac{\partial h}{\partial y_{2}} \rho\right)-\frac{\partial}{\partial h}\left\{\left(-\varepsilon^{2} \tilde{f}\left(y_{1}, \frac{\partial h}{\partial y_{2}}\right) \frac{\partial h}{\partial y_{2}}\right.\right. \\
& \left.\left.+(r-1)\left(\frac{\partial h}{\partial y_{2}}\right)^{2}\left|\frac{\partial h}{\partial y_{2}}\right| \delta\left(y_{1}\right)+\frac{\varepsilon^{2}}{2} \lambda E\left[Y^{2}\right]\right) \rho\right\} \\
& +\frac{1}{2 !} \frac{\partial^{2}}{\partial h^{2}}\left\{\left(\varepsilon^{2}\left(\frac{\partial h}{\partial y_{2}}\right)^{2} \lambda E\left[Y^{2}\right]+\frac{\varepsilon^{4}}{4} \lambda E\left[Y^{4}\right]\right) \rho\right\}
\end{aligned}
$$




$$
\begin{aligned}
& -\frac{1}{3 !} \frac{\partial^{3}}{\partial h^{3}}\left\{\left(\frac{3}{2} \varepsilon^{4}\left(\frac{\partial h}{\partial y_{2}}\right)^{2} \lambda E\left[Y^{4}\right]\right) \rho\right\}+\frac{1}{4 !} \\
& \cdot \frac{\partial^{4}}{\partial h^{4}}\left\{\varepsilon^{4}\left(\frac{\partial h}{\partial y_{2}}\right)^{4} \lambda E\left[Y^{4}\right] \rho\right\}+O\left(\varepsilon^{5}\right) .
\end{aligned}
$$

The conditional probability density $\rho\left(y_{1} \mid h, t\right)$ given by Stratonovich [45] is

$$
\rho\left(y_{1} \mid h, t\right)= \begin{cases}\frac{\sqrt{k}}{2 \pi \sqrt{2 h-k y_{1}^{2}}} & \text { for } k y_{1}^{2}<2 h, \\ 0 & \text { for } k y_{1}^{2}>2 h .\end{cases}
$$

By means of the formulation $\rho\left(y_{1} \mid h, t\right)=\rho\left(y_{1}, h, t\right) / \rho(h, t)$ and integrating (18) with respect to $y_{1}$, the following averaged generalized FPK equation for probability density $\rho(h, t)$ is obtained:

$$
\begin{aligned}
& \frac{\partial \rho(h, t)}{\partial t} \\
& =\frac{\partial}{\partial h}\left\{\left(\varepsilon^{2} F(h)+G(h)-\frac{\varepsilon^{2}}{2} \lambda E\left[Y^{2}\right]\right) \rho(h, t)\right\} \\
& +\frac{1}{2 !} \frac{\partial^{2}}{\partial h^{2}}\left\{\left(\varepsilon^{2} \lambda E\left[Y^{2}\right] h+\frac{\varepsilon^{4}}{4} \lambda E\left[Y^{4}\right]\right) \rho(h, t)\right\} \\
& \quad-\frac{1}{3 !} \frac{\partial^{3}}{\partial h^{3}}\left\{\frac{3 \varepsilon^{4}}{2} h \lambda E\left[Y^{4}\right] \rho(h, t)\right\} \\
& +\frac{1}{4 !} \frac{\partial^{4}}{\partial h^{4}}\left\{\frac{3 \varepsilon^{4}}{2} h^{2} \lambda E\left[Y^{4}\right] \rho(h, t)\right\}+O\left(\varepsilon^{5}\right)
\end{aligned}
$$

where

$$
\begin{aligned}
F(h) & =\oint \frac{\sqrt{k}}{2 \pi} \tilde{f}\left(y_{1}, \frac{\partial h}{\partial y_{2}}\right) d y_{1} \\
G(h) & =\oint-(r-1)\left(\frac{\partial h}{\partial y_{2}}\right)\left|\frac{\partial h}{\partial y_{2}}\right| \delta\left(y_{1}\right) \frac{\sqrt{k}}{2 \pi} d y_{1} \\
& =-\frac{2 \sqrt{k}(r-1)}{\pi} h .
\end{aligned}
$$

It is worth noting that the impulsive damping term should be averaged over a half period since there have two impacts in each period (see Figure 3).

3.2. Approximately Stationary Solution. The averaged generalized FPK (20) is too complicated to be solved analytically. Herein, we seek the approximately stationary solutions. Then, the left-hand side of (20) vanishes and the original equation can be rewritten as

$$
\frac{d J(h)}{d h}=0,
$$

where

$$
\begin{aligned}
J(h)= & \varepsilon^{2} F(h) \rho(h)+G(h) \rho(h) \\
& +\frac{\varepsilon^{2} \lambda E\left[Y^{2}\right]}{2} h \frac{d \rho(h)}{d h}+\frac{\varepsilon^{4} \lambda E\left[Y^{4}\right]}{8} h \frac{d^{2} \rho(h)}{d h^{2}} \\
& +\frac{\varepsilon^{4} \lambda E\left[Y^{4}\right]}{16} \frac{d^{3} \rho(h)}{d h^{3}}+o\left(\varepsilon^{5}\right) .
\end{aligned}
$$

It is easy to know from (23) that $J(h)$ is a constant. In another way, the probability current function satisfies $\left.J(h)\right|_{h \rightarrow \infty}=0$. Thus, we only need to solve the following equation to obtain the approximately stationary solutions:

$$
\begin{aligned}
& \varepsilon^{2} F(h) \rho(h)+G(h) \rho(h)+\frac{\varepsilon^{2} \lambda E\left[Y^{2}\right]}{2} h \frac{d \rho(h)}{d h} \\
& +\frac{\varepsilon^{4} \lambda E\left[Y^{4}\right]}{8} h \frac{d^{2} \rho(h)}{d h^{2}}+\frac{\varepsilon^{4} \lambda E\left[Y^{4}\right]}{16} \frac{d^{3} \rho(h)}{d h^{3}} \\
& +o\left(\varepsilon^{5}\right)=0 .
\end{aligned}
$$

The above equation can be solved by a perturbation technique [43]. The perturbation solution is assumed to be of the form

$$
\rho(h)=\rho_{0}(h)+\varepsilon^{2} \rho_{1}(h)+\varepsilon^{4} \rho_{2}(h)+\cdots,
$$

where $\rho(h)$ satisfies the boundary condition:

$$
\begin{gathered}
\rho(h) \geq 0, \quad h \in(0, \infty), \\
\rho \longrightarrow 0, \quad h \longrightarrow \infty .
\end{gathered}
$$

Substituting (26) into (25) yields the following equations:

$$
\begin{gathered}
F(h) \rho_{0}(h)+\frac{G(h)}{\varepsilon^{2}} \rho_{0}(h)+\frac{\lambda E\left[Y^{2}\right]}{2} h \frac{d \rho_{0}(h)}{d h}=0, \\
F(h) \rho_{1}(h)+\frac{G(h)}{\varepsilon^{2}} \rho_{1}(h)+\frac{\lambda E\left[Y^{2}\right]}{2} h \frac{d \rho_{1}(h)}{d h} \\
=-\frac{\lambda E\left[Y^{4}\right]}{8}\left(h \frac{d^{2} \rho_{0}(h)}{d h^{2}}+\frac{h^{2}}{2} \frac{d^{3} \rho_{0}(h)}{d h^{3}}\right), \ldots
\end{gathered}
$$

Generally speaking, the approximately stationary solution of $\rho(h)$ can be obtained analytically and works well with the numerical simulation results up to order of $\varepsilon^{4}$. The higher terms can be neglected since their effects are relatively small.

The joint PDF for the variables $y_{1}$ and $y_{2}$ can be further obtained as

$$
\begin{aligned}
\rho\left(y_{1}, y_{2}\right) & =\rho(h) \rho\left(y_{1} \mid h\right) y_{2} \\
& =\left.\frac{\sqrt{k}}{2 \pi} \rho(h)\right|_{h=(1 / 2) y_{2}^{2}+(1 / 2) k y_{1}^{2}} .
\end{aligned}
$$

Using the inverse transformation of formula (6), the joint PDF for original displacement $x_{1}$ and velocity $x_{2}$ is calculated as

$$
\begin{aligned}
\rho\left(x_{1}, x_{2}\right) & =\rho\left(y_{1}, y_{2}\right)\left|\frac{\partial\left(y_{1}, y_{2}\right)}{\partial\left(x_{1}, x_{2}\right)}\right| \\
& =\rho\left(y_{1}, y_{2}\right)+\rho\left(-y_{1},-y_{2}\right)=2 \rho\left(y_{1}, y_{2}\right) .
\end{aligned}
$$


Thus, the marginal PDFs for displacement $x_{1}$ and velocity $x_{2}$ are easy to be derived, respectively:

$$
\begin{aligned}
& \rho\left(x_{1}\right)=\int_{-\infty}^{+\infty} \rho\left(x_{1}, s\right) d s, \\
& \rho\left(x_{2}\right)=\int_{-\infty}^{0} \rho\left(s, x_{2}\right) d s .
\end{aligned}
$$

\section{Illustrative Examples}

In this section, two examples are shown to illustrate the proposed procedure. In order to compare the analytical results and numerical results, the impulses magnitudes $Y$ of Poisson white noise are assumed to be Gaussian distributed with zero mean. Thus, an interesting result can be used to simplify the practical computation.

$$
E\left[Y^{4}\right]=3\left(E\left[Y^{2}\right]\right)^{2}
$$

4.1. Rayleigh Oscillator. In this example, a typical Rayleigh vibroimpact system under external Poisson white noise excitation is considered:

$$
\begin{aligned}
\ddot{x}+\varepsilon^{2}\left(\dot{x}^{2}-1\right) \dot{x}+k x & =\varepsilon \xi(t), \quad x<0, \\
\dot{x}_{+} & =-r \dot{x}_{-}, \quad x=0 .
\end{aligned}
$$

In this case, $f(x, \dot{x})=\left(\dot{x}^{2}-1\right) \dot{x}$. The following transformed equation without velocity jump is obtained by means of the Zhuravlev transformation and Dirac delta function.

$$
\begin{aligned}
\ddot{y}+ & \varepsilon^{2}\left(\dot{y}^{2}-1\right) \dot{y}+k y-(r-1) \dot{y}|\dot{y}| \delta(y) \\
& =\varepsilon \operatorname{sgn} y \xi(t) .
\end{aligned}
$$

The corresponding Itô stochastic differential equation is derived as

$$
\begin{aligned}
d y_{1}= & \frac{\partial H}{\partial y_{2}} d t \\
d H= & -\varepsilon^{2}\left(\left(\frac{\partial H}{\partial y_{2}}\right)^{2}-1\right)\left(\frac{\partial H}{\partial y_{2}}\right)^{2} d t \\
& +(r-1)\left(\frac{\partial H}{\partial y_{2}}\right)^{2}\left|\frac{\partial H}{\partial y_{2}}\right| \delta\left(y_{1}\right) d t \\
& +\varepsilon \operatorname{sgn} y_{1} \frac{\partial H}{\partial y_{2}} a d C(t)+\frac{\varepsilon^{2}}{2}[d C(t)]^{2} .
\end{aligned}
$$

According to (20) and (21), $F(h)$ is calculated as follows in the averaged generalized FPK equation for probability density $\rho(h, t)$ :

$$
F(h)=\oint-\frac{\sqrt{k}}{2 \pi} \beta\left(\left(\frac{\partial h}{\partial y_{2}}\right)^{2}-1\right) \frac{\partial h}{\partial y_{2}} d y_{1}=\frac{3}{2} h^{2}-h .
$$

Using the perturbation technique, the approximately stationary solution for energy PDF is obtained:

$$
\begin{aligned}
& \rho(h) \\
& =\widetilde{C} e^{-N}\left(1+\varepsilon^{2}\left(D h+E h^{2}+F h^{3}+G h^{4}+M h^{5}\right)\right),
\end{aligned}
$$

where

$$
\begin{aligned}
A & =\lambda E\left[Y^{2}\right], \\
B & =\lambda E\left[Y^{4}\right], \\
C & =-1-\frac{2(r-1) \sqrt{k}}{\pi \varepsilon^{2}}, \\
D & =\frac{3 B}{4 A^{2}}-\frac{B C^{2}}{A^{3}}, \\
E & =\frac{B C^{3}}{2 A^{4}}-\frac{21 B C}{8 A^{3}}, \\
F & =\frac{3 B C^{2}}{2 A^{4}}-\frac{15 B}{8 A^{3}}, \\
G & =\frac{27 B C}{16 A^{4}}, \\
M & =\frac{27 B}{40 A^{4}}, \\
N & =\frac{2}{A}\left(\frac{3 h^{2}}{4}+C h\right) .
\end{aligned}
$$

Furthermore, the joint PDF for original displacement $x_{1}$ and velocity $x_{2}$ can be calculated by substituting (37) and (38) into (29) and (30) as

$$
\rho\left(x_{1}, x_{2}\right)=\left.\frac{\sqrt{k}}{\pi} \rho(h)\right|_{h=(1 / 2) y_{2}^{2}+(1 / 2) k y_{1}^{2}} .
$$

To verify the effectiveness of the proposed procedure, analytical results and Monte-Carlo simulation results are shown in Figure 4 for PDFs of system energy with $k=1, r=$ $0.99, \lambda=1$, and $E\left[Y^{2}\right]=1$ and two different values of small parameter $\varepsilon(\varepsilon=0.1$ and 0.2$)$. The solid line is the analytical results obtained by the proposed method while $\bullet$ represents the Monte-Carlo simulation results based on the original system (33a) and (33b). It is obvious that the two results agree well with each other. In order to illustrate the effects of average arrival rate of external Poisson white noise on the accuracy of the approximately stationary solution, Figure 5 shows PDFs of system energy analytically and numerically with different values of $\lambda(\lambda=1$ and 2$)$ when $\varepsilon$ is fixed as 0.1 . It is seen that the proposed method works well.

To investigate the effects of restitution coefficient on the stationary response of vibroimpact system, numerical and analytical results are shown in Figures 6-8 with the parameters $k=1, \varepsilon=0.1, \lambda=1$, and $E\left[Y^{2}\right]=1$ and different values of $r$. It is seen from Figure 6 that the stationary PDFs of total energy are shown for $r=0.97,0.98,0.99$, and 0.995 . The PDFs of total energy present lower peaks with the increase of restitution coefficient. It is found that the values of peak are at the system's static equilibrium position when $r=0.97$, 0.98 while they do not stay there when $r=0.99,0.995$. This may mean that the steady state has a qualitative change with the increase of restitution coefficient. To show it more clearly, Figures 7 and 8 provide analytical and numerical results of 


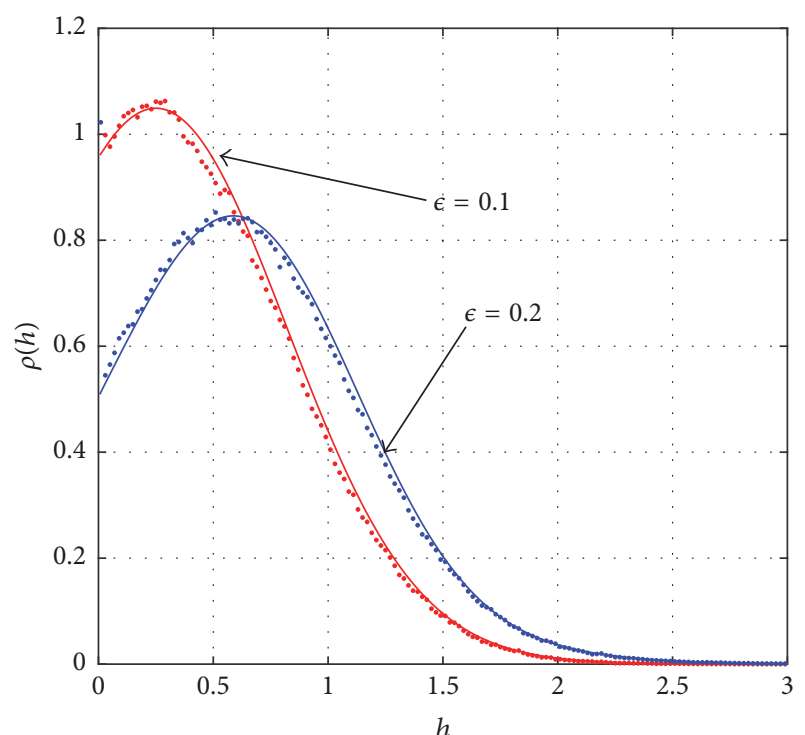

FIGURE 4: Stationary PDFs of total energy obtained numerically and analytically for different values of small parameter $\varepsilon$.

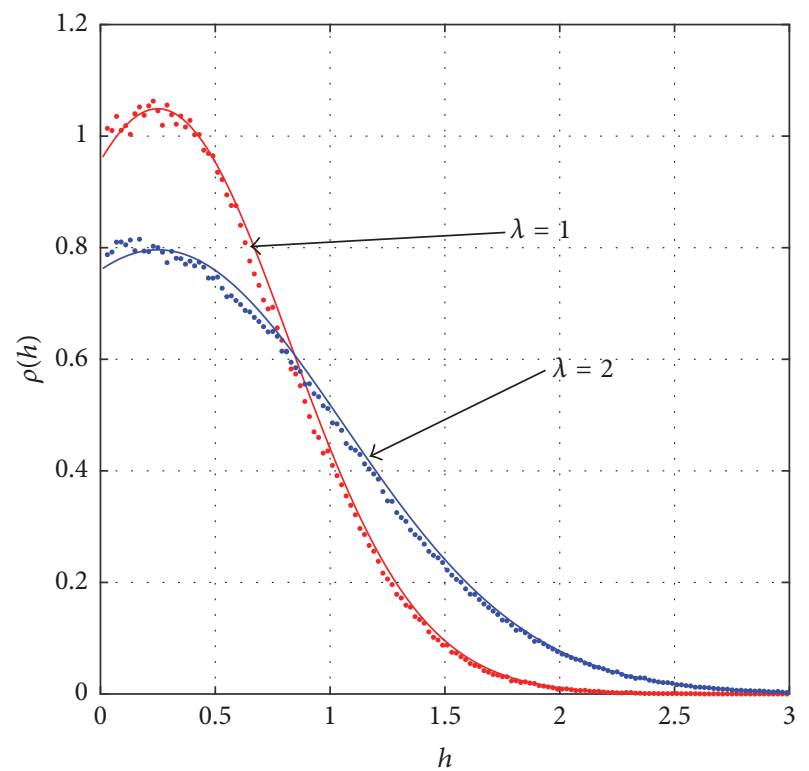

FIGURE 5: Stationary PDFs of total energy obtained numerically and analytically for different values of average arrival rate $\lambda$.

joint PDFs of the displacement $x_{1}$ and velocity $x_{2}$ for $r=0.98$ and $r=0.995$, respectively. It is seen that the analytical results agree well with the numerical results. In Figure 7 , the joint PDFs of the displacement $x_{1}$ and velocity $x_{2}$ have a singular peak which appears at the static equilibrium position. When $r$ increases to $r=0.995$, the joint PDFs present the shape of crater in Figure 8. This illuminates the occurrence of Pbifurcation according to the concept of stochastic bifurcation. In other words, the restitution coefficient $r$ can induce the occurrence of P-bifurcation.

4.2. Van der Pol Oscillator. As an example, a typical Van der Pol vibroimpact system under external Poisson white noise excitation is considered:

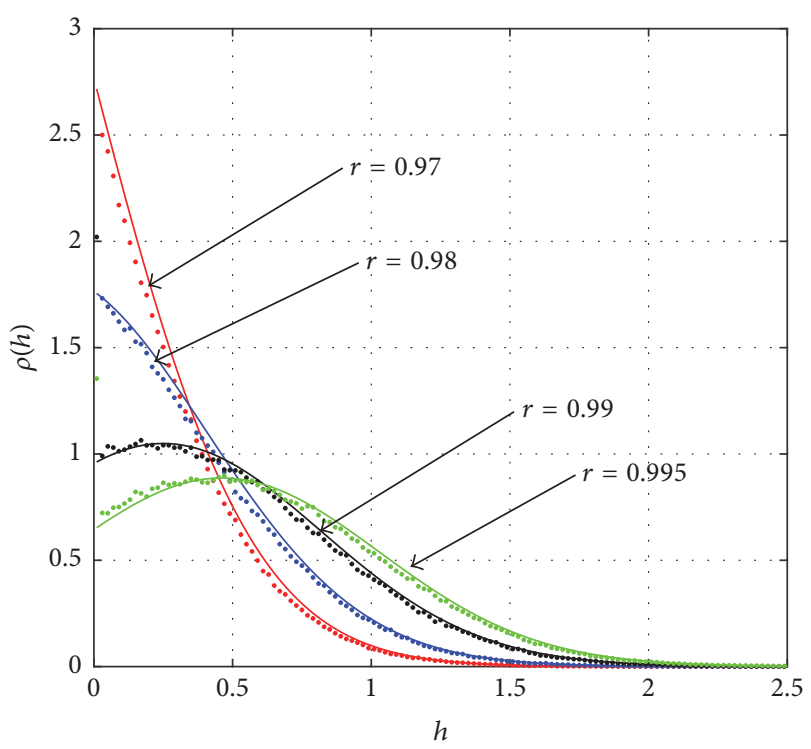

FIGURE 6: Stationary PDFs of total energy obtained numerically and analytically for different values of restitution coefficient $r$.

$$
\begin{aligned}
\ddot{x}-\varepsilon^{2} \beta\left(1-x^{2}\right) \dot{x}+k x & =\varepsilon \xi(t), \quad x<0, \\
\dot{x}_{+} & =-r \dot{x}_{-}, \quad x=0 .
\end{aligned}
$$

Based on (9), the transformed system without velocity is obtained as

$$
\begin{aligned}
\ddot{y}- & \varepsilon^{2} \beta\left(1-y^{2}\right) \dot{y}+k y-(r-1) \dot{y}|\dot{y}| \delta(y) \\
& =\varepsilon \operatorname{sgn} y \xi(t) .
\end{aligned}
$$

The Itô stochastic differential equation about displacement $y_{1}$ and energy $H$ is derived as

$$
\begin{aligned}
d y_{1}= & \frac{\partial H}{\partial y_{2}} d t \\
d H= & \varepsilon^{2} \beta\left(1-y_{1}^{2}\right)\left(\frac{\partial H}{\partial y_{2}}\right)^{2} d t \\
& +(r-1)\left(\frac{\partial H}{\partial y_{2}}\right)^{2}\left|\frac{\partial H}{\partial y_{2}}\right| \delta\left(y_{1}\right) d t \\
& +\varepsilon \operatorname{sgn} y_{1} \frac{\partial H}{\partial y_{2}} d C(t)+\frac{\varepsilon^{2}}{2}[d C(t)]^{2} .
\end{aligned}
$$

In the averaged generalized FPK $(20), F(h)$ here is calculated as

$$
F(h)=\oint-\frac{\sqrt{k}}{2 \pi} \beta\left(1-y_{1}^{2}\right) \frac{\partial h}{\partial y_{2}} d y_{1}=\frac{\beta}{2 k} h^{2}-\beta h .
$$




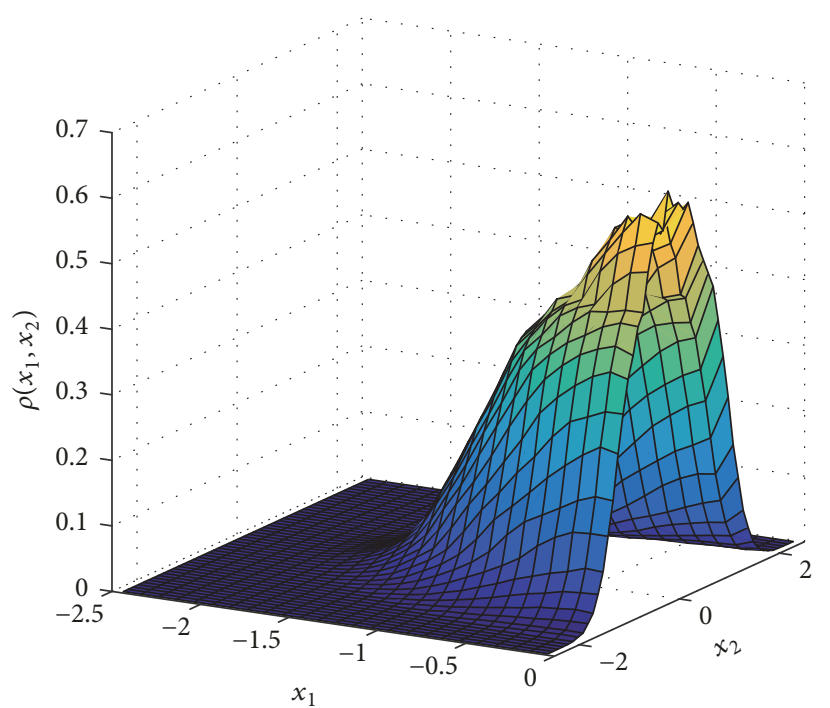

(a)

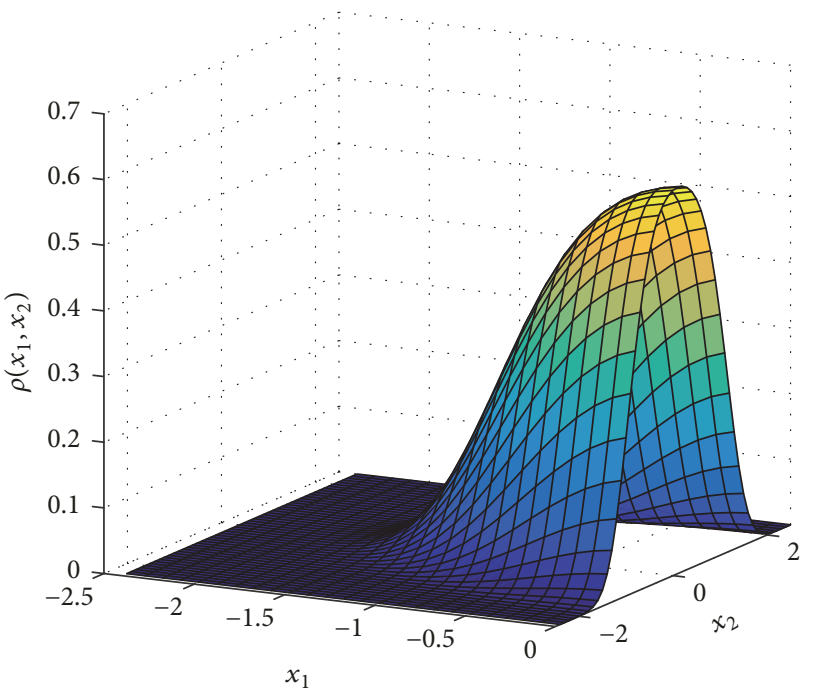

(b)

FIGURE 7: Joint PDFs of the displacement $x_{1}$ and velocity $x_{2}$ for $r=0.98$ : (a) Monte-Carlo results; (b) analytical results.

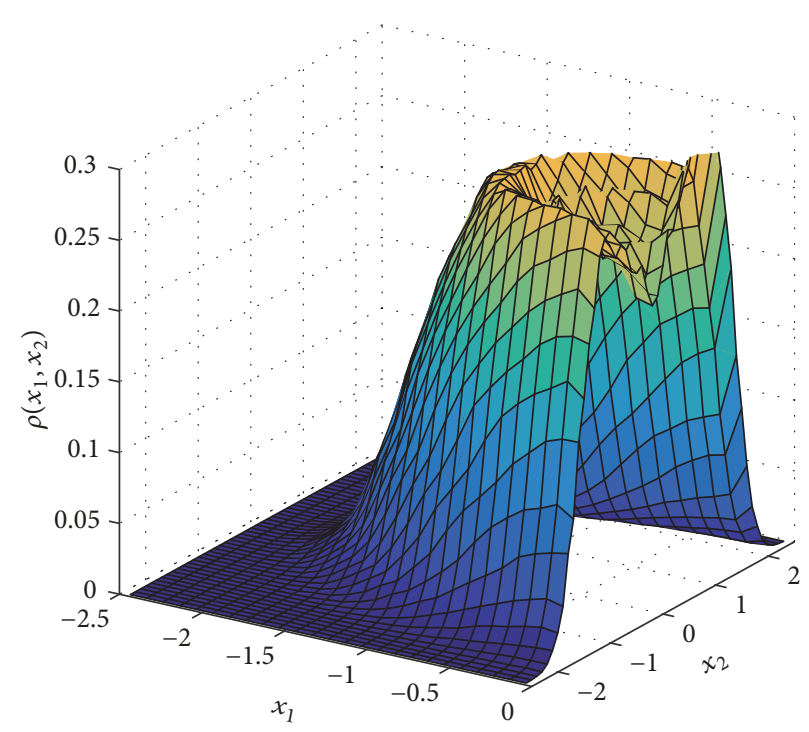

(a)

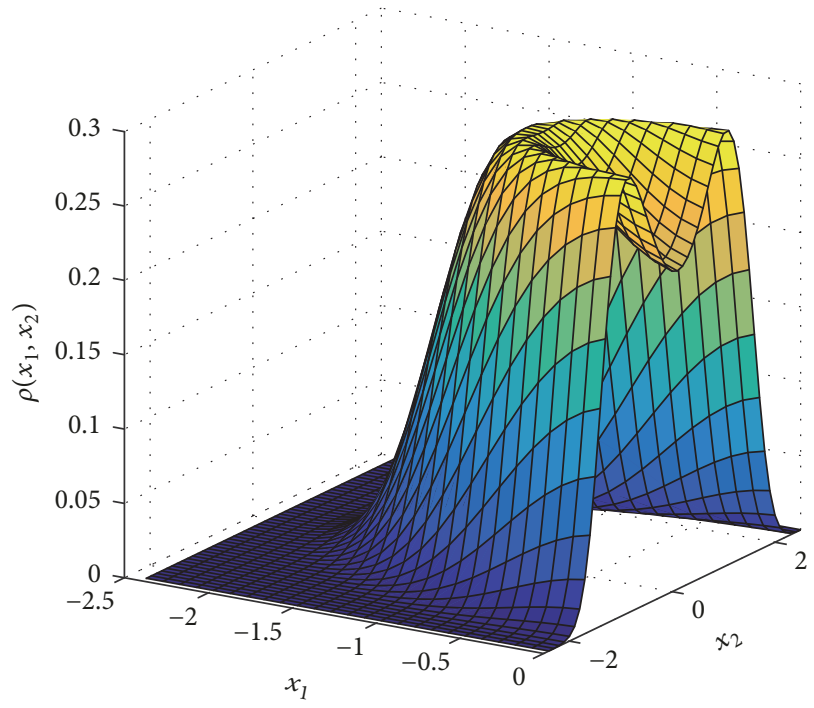

(b)

FIGURE 8: Joint PDFs of the displacement $x_{1}$ and velocity $x_{2}$ for $r=0.995$ : (a) Monte-Carlo results; (b) analytical results.

Thus, $\rho(h)$ is obtained analytically by solving the corresponding averaged generalized FPK equation as

$$
\begin{aligned}
& \rho(h) \\
& \quad=\widetilde{C} e^{-N}\left(1+\varepsilon^{2}\left(D h+E h^{2}+F h^{3}+G h^{4}+M h^{5}\right)\right),
\end{aligned}
$$

where

$$
\begin{aligned}
& A=\lambda E\left[Y^{2}\right], \\
& B=\lambda E\left[Y^{4}\right],
\end{aligned}
$$

$C=-\beta-\frac{2(r-1) \sqrt{k}}{\pi \varepsilon^{2}}$

$D=\frac{B \beta}{4 A^{2} k}-\frac{B C^{2}}{A^{3}}$,

$E=\frac{B C^{3}}{2 A^{4}}-\frac{7 B C \beta}{8 A^{3} k}$,

$F=\frac{B C^{2} \beta}{2 A^{4} k}-\frac{5 B \beta^{2}}{24 A^{3} k^{2}}$,

$G=\frac{3 B C \beta^{2}}{16 A^{4} k^{2}}$, 


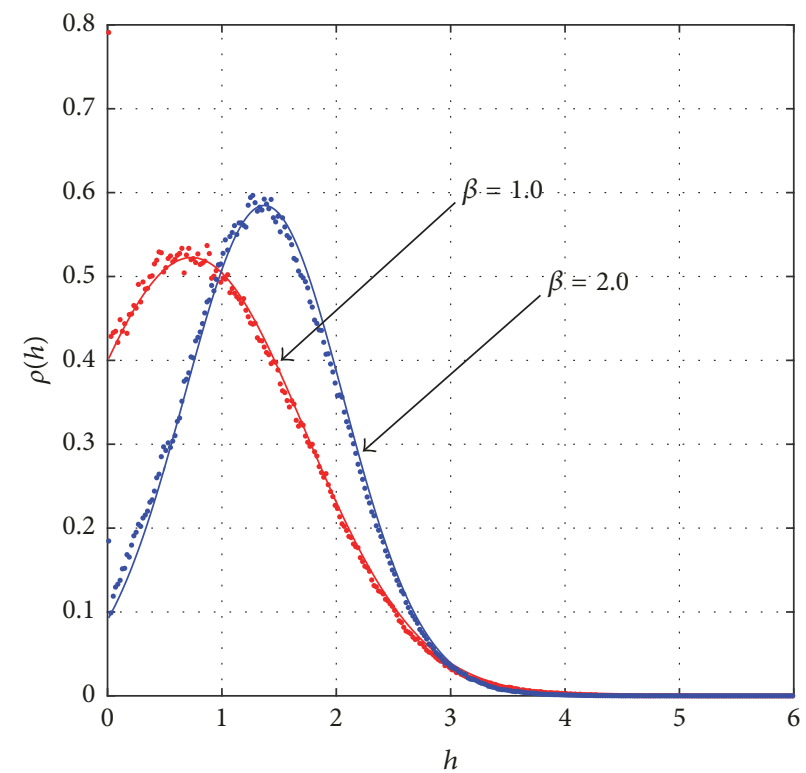

FIGURE 9: Stationary PDFs of total energy obtained numerically and analytically for different values of $\beta$.

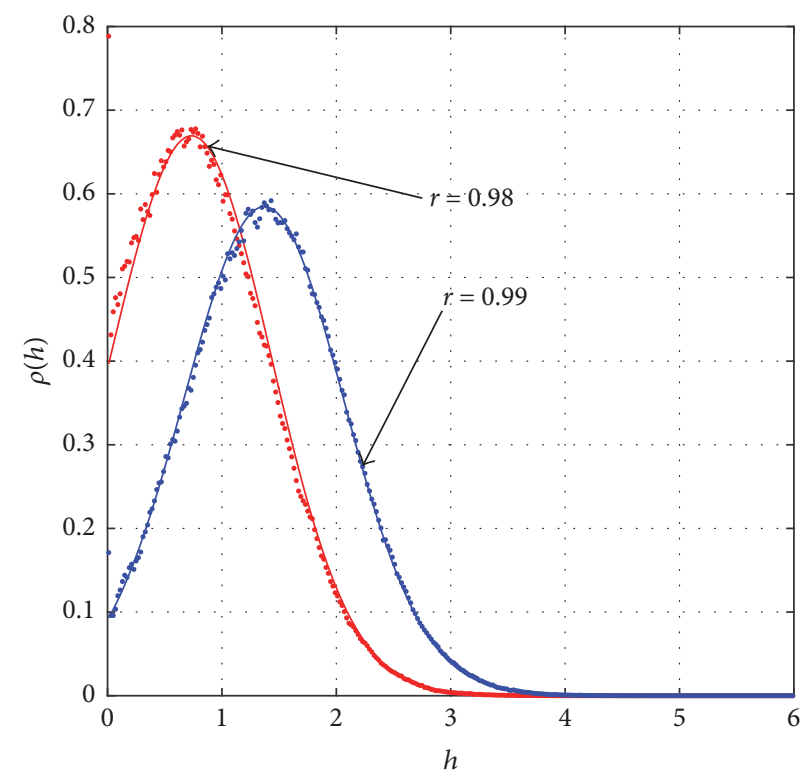

FIGURE 10: Stationary PDFs of total energy obtained numerically and analytically for different values of restitution coefficient $r$.

$$
\begin{aligned}
& M=\frac{B \beta^{3}}{40 A^{4} k^{3}}, \\
& N=\frac{2}{A}\left(\frac{\beta h^{2}}{4 k}+C h\right) .
\end{aligned}
$$

Furthermore, the PDFs $\rho\left(x_{1}, x_{2}\right), \rho\left(x_{1}\right)$, and $\rho\left(x_{2}\right)$ can be calculated following (29)-(31), respectively.

To demonstrate the effectiveness of the proposed method, numerical and analytical results of response PDFs are provided in Figures 9-13. The effects of damping coefficient on the accuracy of the energy PDFs are shown in Figure 9 with $\varepsilon=0.1, r=0.99, \lambda=1$, and $E\left[Y^{2}\right]=1$ and two different values of $\beta(\beta=1.0$ and 2.0). Stationary PDFs of total energy obtained numerically and analytically for different values of restitution coefficient $r$ are presented in Figure 10. It is clear from Figures 9 and 10 that the analytical results give powerful validation with Monte-Carlo simulation results. The joint PDFs of the displacement $x_{1}$ and velocity $x_{2}$ for different impulse intensities are shown numerically and analytically in Figures 11 and 12. The corresponding marginal PDFs $\rho\left(x_{1}\right)$ and $\rho\left(x_{2}\right)$ are provided in Figures 13(a) and 13(b), respectively. It is seen that the results obtained by using the proposed method and those from Monte-Carlo simulation are in good agreement. Additionally, the increase of impulse intensity 


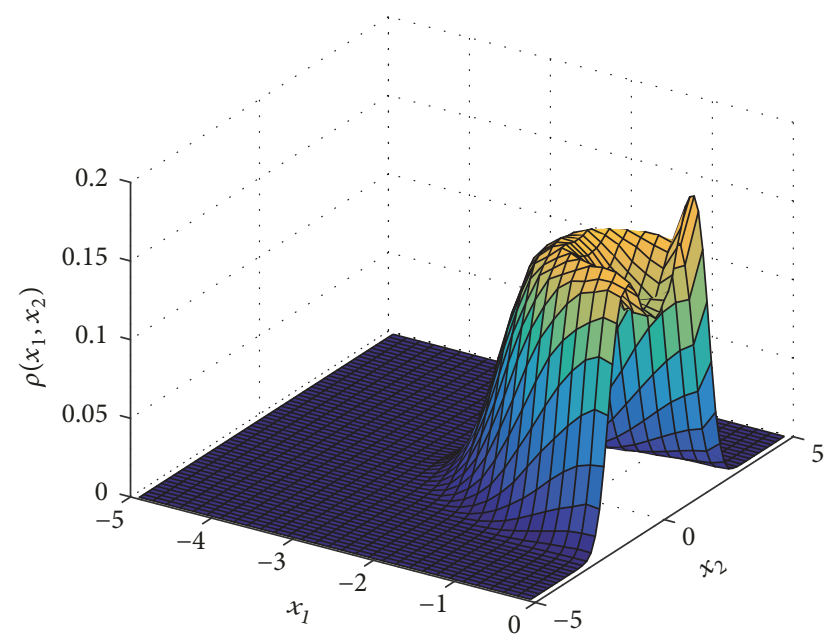

(a)

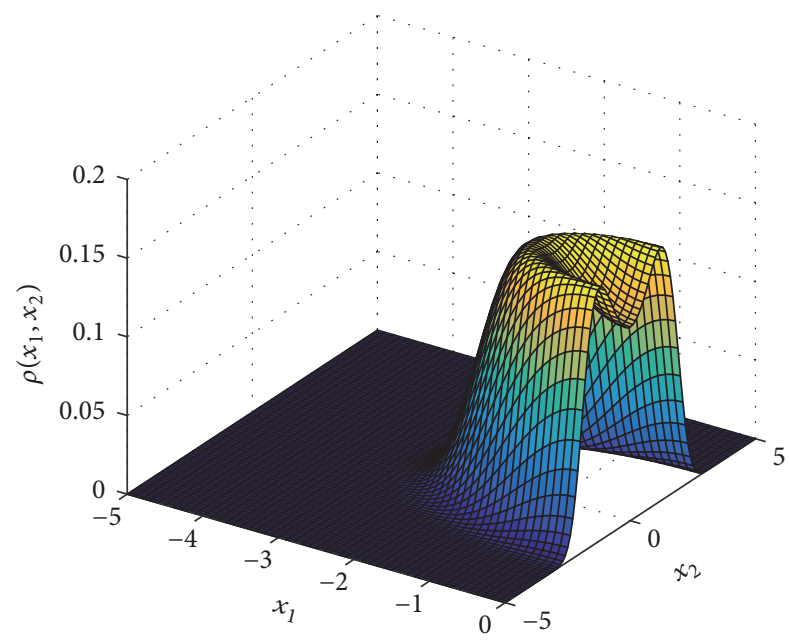

(b)

FIGURE 11: Joint PDFs of the displacement $x_{1}$ and velocity $x_{2}$ for $E\left[Y^{2}\right]=1.0$ : (a) Monte-Carlo results; (b) analytical results.

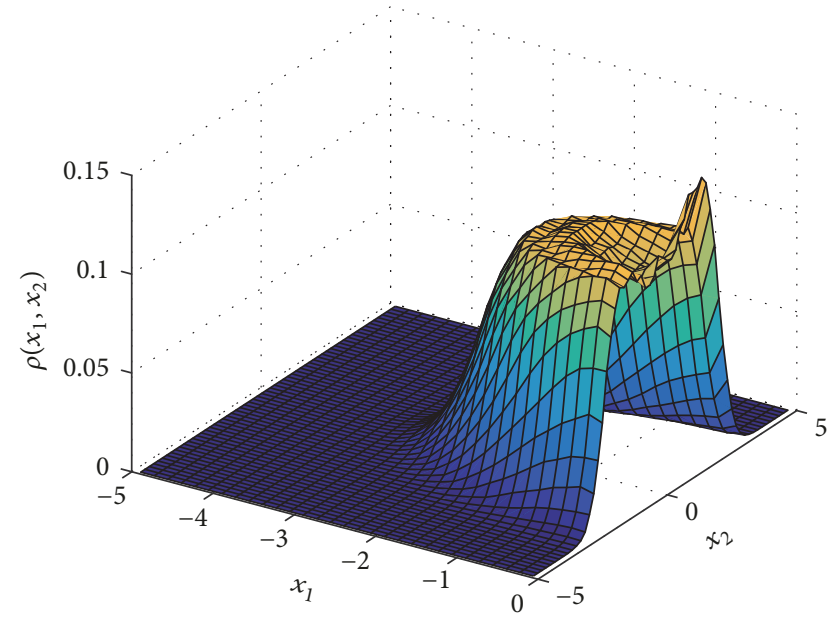

(a)

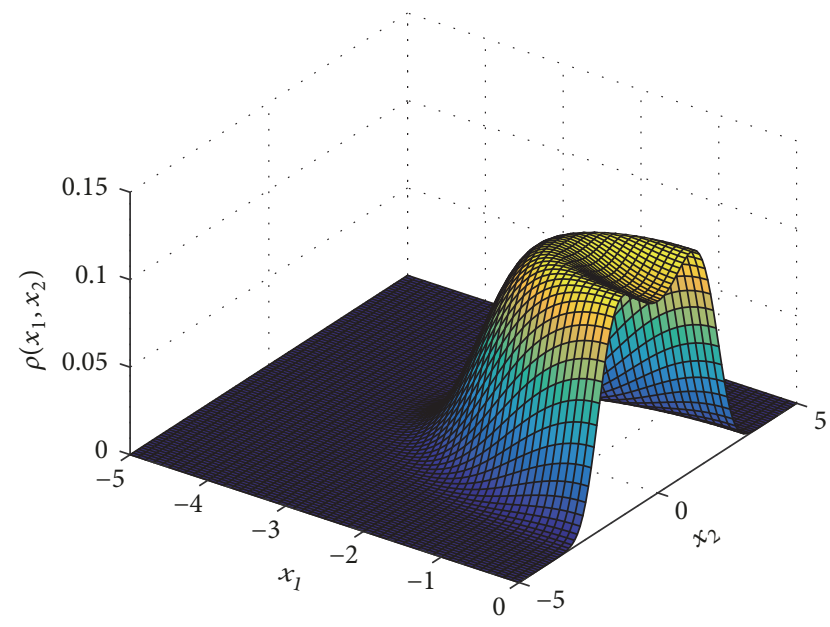

(b)

FIgURE 12: Joint PDFs of the displacement $x_{1}$ and velocity $x_{2}$ for $E\left[Y^{2}\right]=2.0$ : (a) Monte-Carlo results; (b) analytical results.

only effects the values of peaks of stationary response PDFs, and there is no occurrence of P-bifurcation.

\section{Conclusions}

The paper is devoted to presenting a solution procedure for predicting stationary responses of single-degree-of-freedom lightly nonlinear vibroimpact systems with inelastic impact subjected to external Poisson white noise excitations. The classical model with instantaneous impacts is used and the rigid barrier is located at the static equilibrium of the system. The solution procedure consists of three steps. First, the Zhuravlev nonsmooth coordinate transformation and the Dirac delta function are adopted to convert the original vibroimpact system to a new system without velocity jump. Second, the Itô stochastic differential equations with correction terms are obtained and the averaged generalized FokkerPlanck-Kolmogorov (FPK) equation is derived by stochastic averaging method. Third, the perturbation method is utilized to solve the averaged generalized FPK equation and the inverse transformation of the Zhuravlev nonsmooth coordinate transformation is used to establish the PDFs for original vibroimpact system. Furthermore, two examples are presented to show the effectiveness of the proposed method. It is found that the analytical results obtained by the proposed method and the Monte-Carlo simulation results agree well. Therefore, the proposed procedure is quite promising and can be extended to more vibroimpact systems subject to Poisson white noise excitation. 


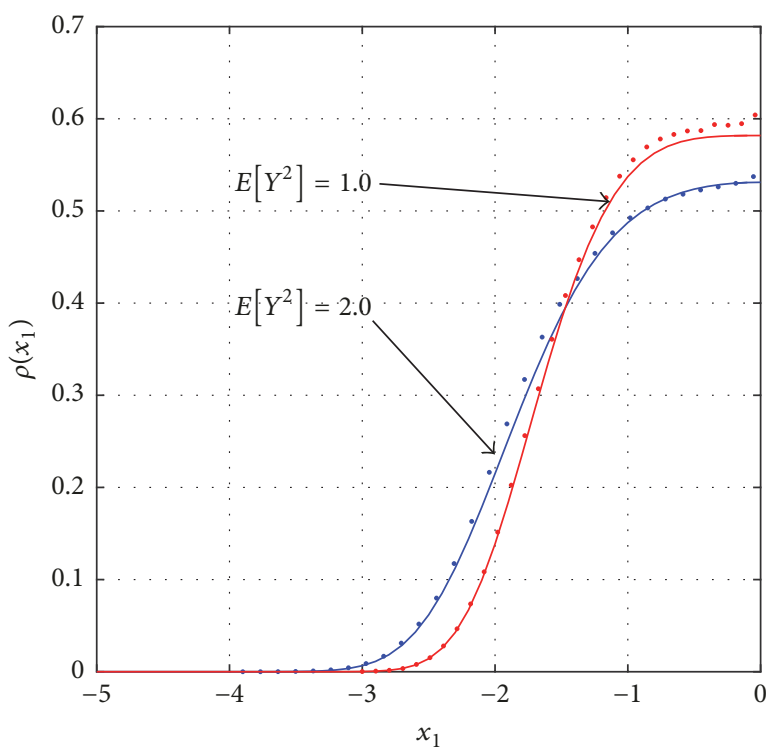

(a)

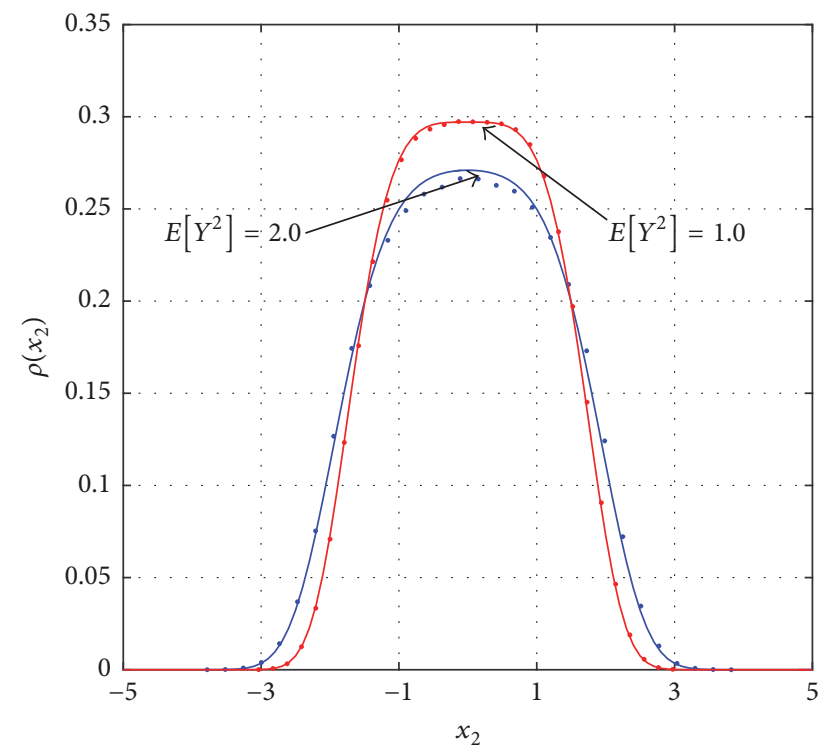

(b)

FIGURE 13: Stationary PDFs obtained numerically and analytically for different values of random impulse intensity $E\left[Y^{2}\right]$. -, analytical results; , Monte-Carlo results: (a) PDFs of displacement $x_{1}$; (b) PDFs of velocity $x_{2}$.

\section{Conflicts of Interest}

The authors declare that they have no conflicts of interest.

\section{Acknowledgments}

This work was supported by the National Natural Science Foundation of China (Grant nos. 11702200, 11702214, and 11702201) and the Fundamental Research Funds for the Central Universities (Grant no. JB170705).

\section{References}

[1] A. I. Raouf, Vibro-impact Dynamics: Modeling, Mapping and Applications, vol. 43 of Lecture Notes in Applied and Computational Mechanics, Springer, Berlin, Germany, 2009.

[2] V. I. Babitsky, Theory of vibro-impact systems and applications, Foundations of Engineering Mechanics, Springer-Verlag, Berlin, Germany, 1998.

[3] B. Brogliato, Nonsmooth Mechanics: Models, Dynamics and Control, Springer, London, UK, 1999.

[4] A. L. Paget, "Vibration in steam turbine buckets and damping by impacts," Engineering, vol. 143, pp. 305-307, 1937.

[5] L. Kop, "Wear of power plant components due to impact and sliding," Applied Mechanics Reviews, vol. 50, p. 387, 1997.

[6] M. F. Dimentberg and D. V. Iourtchenko, "Random vibrations with impacts: a review," Nonlinear Dynamics, vol. 36, no. 2-4, pp. 229-254, 2004.

[7] R. A. Ibrahim, "Recent advances in vibro-impact dynamics and collision of ocean vessels," Journal of Sound and Vibration, vol. 333, no. 23, pp. 5900-5916, 2014.

[8] B. Chiaia, O. Kumpyak, L. Placidi, and V. Maksimov, "Experimental analysis and modeling of two-way reinforced concrete slabs over different kinds of yielding supports under short-term dynamic loading," Engineering Structures, vol. 96, pp. 88-99, 2015.

[9] U. Andreaus, B. Chiaia, and L. Placidi, "Soft-impact dynamics of deformable bodies," Continuum Mechanics and Thermodynamics, vol. 25, no. 2-4, pp. 375-398, 2013.

[10] S. W. Shaw, "The Dynamics of a Harmonically Excited System Having Rigid Amplitude Constraints, Part 2: Chaotic Motions and Global Bifurcations," Journal of Applied Mechanics, vol. 52, no. 2, pp. 459-464, 1985.

[11] J. Feng and W. Xu, "Analysis of bifurcations for non-linear stochastic non-smooth vibro-impact system via top Lyapunov exponent," Applied Mathematics and Computation, vol. 213, no. 2, pp. 577-586, 2009.

[12] G.-W. Luo and J.-H. Xie, "Hopf bifurcation of a two-degree-offreedom vibro-impact system," Journal of Sound and Vibration, vol. 213, no. 3, pp. 391-408, 1998.

[13] G. J. Li, W. Xu, L. Wang, and J. Q. Feng, "Investigation of the bifurcation of a stochastic van der Pol system," Acta Physica Sinica, vol. 57, no. 4, pp. 2107-2114, 2008.

[14] W. Chin, E. Ott, H. E. Nusse, and C. Grebogi, "Grazing bifurcations in impact oscillators," Physical Review E: Statistical, Nonlinear, and Soft Matter Physics, vol. 50, no. 6, pp. 4427-4444, 1994.

[15] G. W. Luo, Y. D. Chu, Y. L. Zhang, and J. G. Zhang, "Double Neimark-Sacker bifurcation and torus bifurcation of a class of vibratory systems with symmetrical rigid stops," Journal of Sound and Vibration, vol. 298, no. 1-2, pp. 154-179, 2006.

[16] C. Budd and F. Dux, "Chattering and related behaviour in impact oscillators," Philosophical Transactions of the Royal Society of London A, vol. 347, pp. 365-389, 1994.

[17] J. Q. Feng, W. Xu, and Y. J. Niu, "Chattering bifurcations in a duffing unilateral vibro-impact system," Acta Physica Sinica, vol. 59, article 0157, 2010. 
[18] D. J. Wagg, "Periodic sticking motion in a two-degree-of-freedom impact oscillator," International Journal of Non-Linear Mechanics, vol. 40, no. 8, pp. 1076-1087, 2005.

[19] C. Li, W. Xu, L. Wang, and Z. P. Wang, "Impulsive control of sticking motion in van der Pol one-sided constraint system," Applied Mathematics and Computation, vol. 248, pp. 363-370, 2014.

[20] V. Avrutin and M. Schanz, "Border-collision period-doubling scenario," Physical Review E: Statistical, Nonlinear, and Soft Matter Physics, vol. 70, no. 2, Article ID 026222, 2004.

[21] M. F. Dimentberg, tatistical Dynamics of Non-linear and TimeVarying Systems, Mechanical Engineering Research Studies, Research Studies Press, Taunton, UK, 1988.

[22] V. F. Zhuravlev, "A method for analyzing vibration-impact systems by means of special functions," Mechanics of Solids, vol. 11, no. 2, pp. 23-27, 1976.

[23] M. B. Su and H. W. Rong, "Resonance response of a singledegree-of-freedom nonlinear vibro-impact system to a narrowband random parametric excitation," Chinese Physics B, vol. 20, Article ID 060501, 2011.

[24] N. S. Namachchivaya and J. H. Park, "Stochastic dynamics of impact oscillators," Journal of Applied Mechanics, vol. 72, no. 6, pp. 862-870, 2005.

[25] H.-S. Jing and K.-C. Sheu, "Exact stationary solutions of the random response of a single-degree-of-freedom vibro-impact system," Journal of Sound and Vibration, vol. 141, no. 3, pp. 363373, 1990.

[26] H. T. Zhu, "Stochastic response of vibro-impact Duffing oscillators under external and parametric Gaussian white noises," Journal of Sound and Vibration, vol. 333, no. 3, pp. 954-961, 2014.

[27] M. F. Dimentberg, O. Gaidai, and A. Naess, "Random vibrations with strongly inelastic impacts: response PDF by the path integration method," International Journal of Non-Linear Mechanics, vol. 44, no. 7, pp. 791-796, 2009.

[28] M. F. Dimentberg and A. I. Menyailov, "Response of a Singlemass Vibroimpact System to White-noise Random Excitation," ZAMM - Journal of Applied Mathematics and Mechanics, vol. 59, no. 12, pp. 709-716, 1979.

[29] D. V. Iourtchenko and L. L. Song, "Numerical investigation of a response probability density function of stochastic vibroimpact systems with inelastic impacts," International Journal of NonLinear Mechanics, vol. 41, no. 3, pp. 447-455, 2006.

[30] J. Q. Feng, W. Xu, H. W. Rong, and R. Wang, "Stochastic responses of Duffing-Van der Pol vibro-impact system under additive and multiplicative random excitations," International Journal of Non-Linear Mechanics, vol. 44, no. 1, pp. 51-57, 2009.

[31] C. Li, W. Xu, J. Q. Feng, and L. Wang, "Response probability density functions of Duffing-Van der Pol vibro-impact system under correlated Gaussian white noise excitation," Physica A, vol. 392, pp. 1269-1279, 2013, Physica A.

[32] H. T. Zhu, "Response of a vibro-impact Duffing system with a randomly varying damping term," International Journal of NonLinear Mechanics, vol. 65, pp. 53-62, 2014.

[33] Z. L. Huang, Z. H. Liu, and W. Q. Zhu, "Stationary response of multi-degree-of-freedom vibro-impact systems under white noise excitations," Journal of Sound and Vibration, vol. 275, no. 1-2, pp. 223-240, 2004.

[34] M. Xu, Y. Wang, X. Jin, and Z. Huang, "Random vibration with inelastic impact: equivalent nonlinearization technique," Journal of Sound and Vibration, vol. 333, no. 1, pp. 189-199, 2014.
[35] N. Moshchuk and R. A. Ibrahim, "Response statistics of ocean structures to non-linear hydrodynamic loading. Part II: NonGaussian ocean waves," Journal of Sound and Vibration, vol. 191, no. 1, pp. 107-128, 1996.

[36] N. H. Ko, K. P. You, and Y. Kim, "The effect of non-gaussian local wind pressures on a side face of a square building," Journal of Wind Engineering and Industrial Aerodynamics, vol. 93, no. 5, pp. 383-397, 2005.

[37] M. Di Paola and G. Falsone, "Stochastic dynamics of nonlinear systems driven by nonnormal delta-correlated processes," American Society of Mechanical Engineers. Transactions of the ASME. Journal of Applied Mechanics, vol. 60, no. 1, pp. 141-148, 1993.

[38] Y. Zeng and W. Q. Zhu, "Stochastic averaging of n-dimensional non-linear dynamical systems subject to non-Gaussian wideband random excitations," International Journal of Non-Linear Mechanics, vol. 45, no. 5, pp. 572-586, 2010.

[39] Y. Zeng and W. Q. Zhu, "Stochastic averaging of quasi linear systems driven by Poisson white noise," Probabilistic Engineering Mechanics, vol. 2, pp. 99-107, 2010.

[40] C. Proppe, "Stochastic linearization of dynamical systems under parametric Poisson white noise excitation," International Journal of Non-Linear Mechanics, vol. 38, no. 4, pp. 543-555, 2003.

[41] H. T. Zhu, "Stochastic response of a vibro-impact duffing system under external poisson impulses," Nonlinear Dynamics, 2015.

[42] W. Q. Zhu, Nonlinear Stochastic Dynamics and Control: Framework of Hamiltonian Theory, Science Press, Beijing, China, 2003.

[43] G. Q. Cai and Y. K. Lin, "Response distribution of non-linear systems excited by non-Gaussian impulsive noise," International Journal of Non-Linear Mechanics, vol. 27, no. 6, p. 955, 1992.

[44] M. Di Paola and M. Vasta, "Stochastic integro-differential and differential equations of non-linear systems excited by parametric Poisson pulses," International Journal of Non-Linear Mechanics, vol. 32, no. 5, pp. 855-862, 1997.

[45] R. Stratonovich, Topics in the Theory of Random Noise, vol. 1, Gordon and Breach Science Publishers, New York, NY, USA, 1963. 


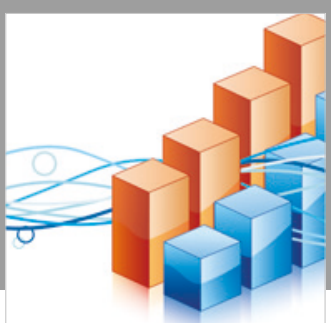

Advances in

Operations Research

\section{-n-m}
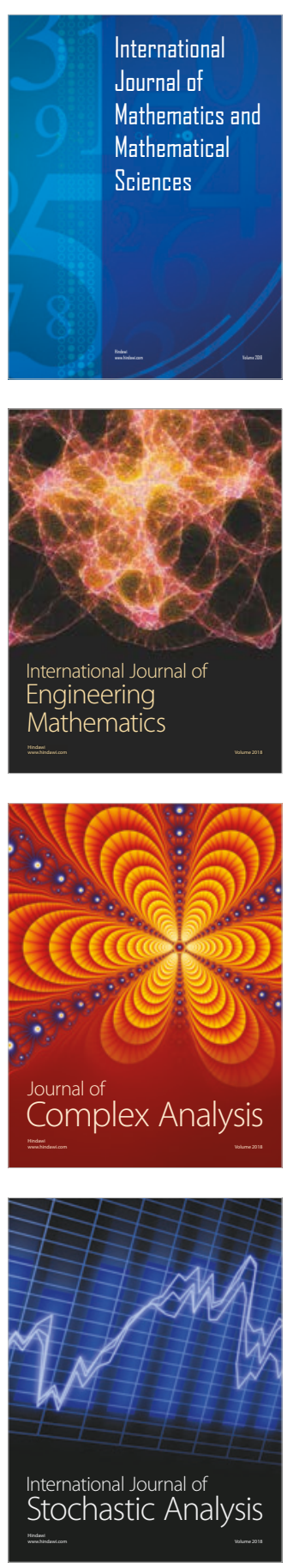
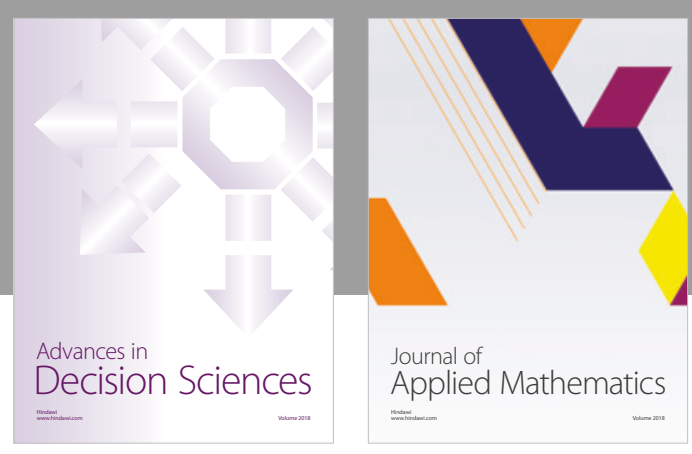

Journal of

Applied Mathematics
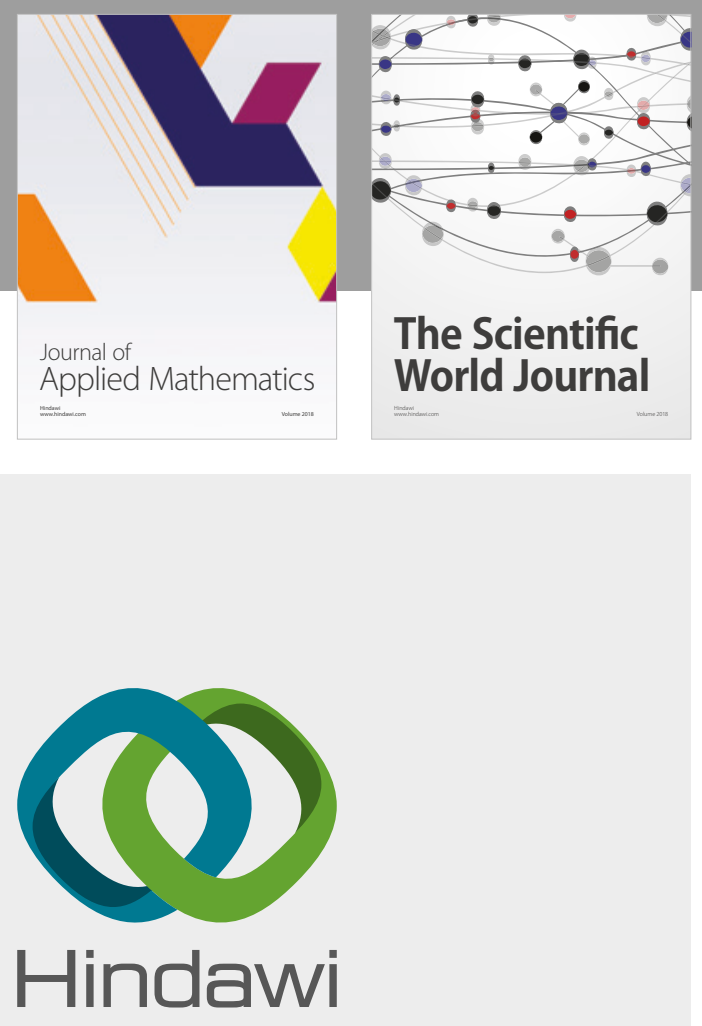

Submit your manuscripts at

www.hindawi.com

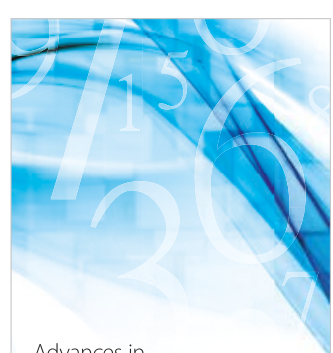

Advances in
Numerical Analysis
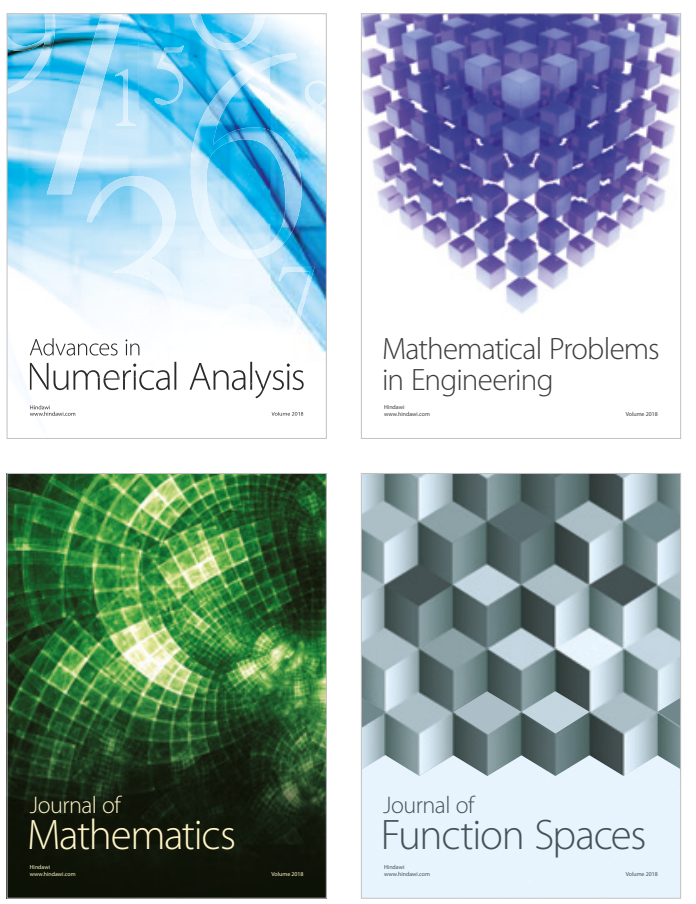

Mathematical Problems in Engineering

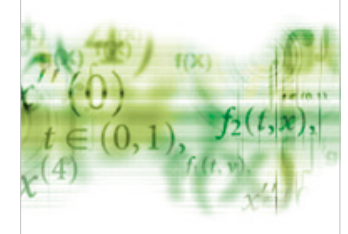

International Journal of

Differential Equations

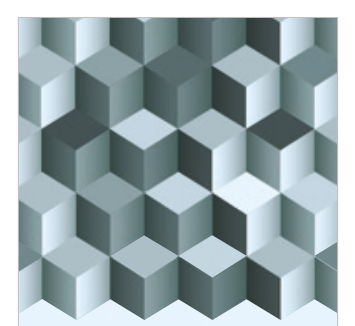

Journal of

Function Spaces

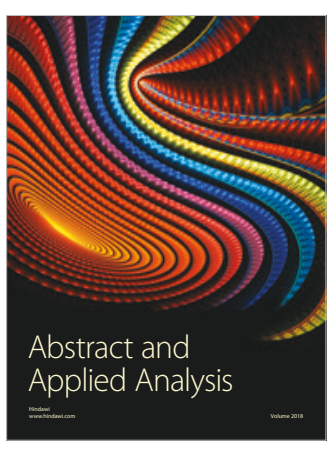

The Scientific

World Journal

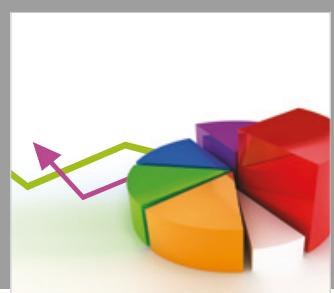

Journal of

Probability and Statistics
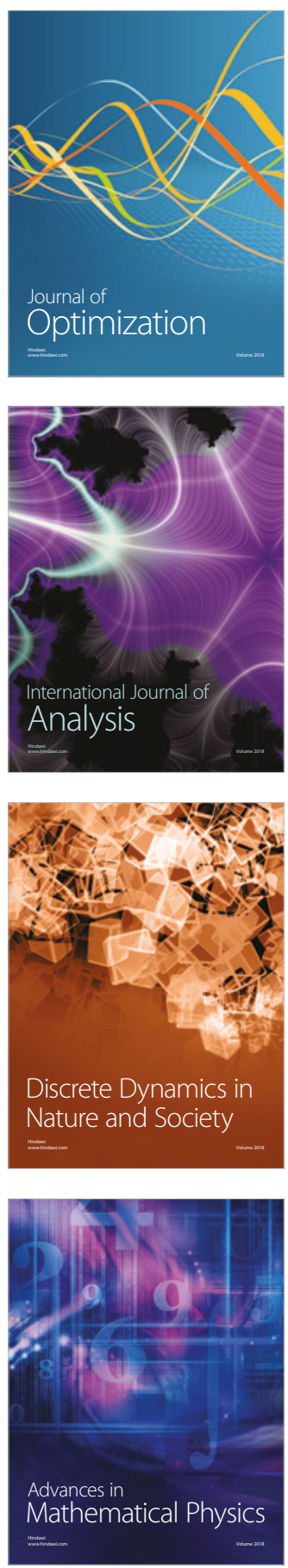